\section{Submitted: 07.04.2019 Accepted: \\ Effect of adding a capsule with activated charcoal to abdominal ultrasound preparation on image quality} 03.10.2019

Published: 31.03.2020

Keywords

pancreas, activated charcoal, improvement

Correspondence: Motaen Tomehy, P.O. Box 1003, Zip code 45142 Jazan, Saudi Arabia; tel.:+966506609787,e-mail:mut315@hotmail.com

DOI: $10.15557 / J o U .2020 .0003$

\author{
Ahmed Abdul Jabar ${ }^{1}$, Ibrahim Abbas², Nabeel Mishah³, \\ Mohammed Wazan', Motaen Tomehy \\ ${ }^{1}$ College of Medicine, King Abdulaziz University, Jeddah, Saudi Arabia \\ ${ }^{2}$ College of Applied Medical Science, King Abdulaziz University, Jeddah, Saudi Arabia \\ ${ }^{3}$ King Abdulaziz University Hospital, Jeddah, Saudi Arabia \\ ${ }^{4} R 4$ resident, Radiology Technologist Fellowship Program, King Abdulaziz, University Hospital, \\ Jeddah, Saudi Arabia
}

\begin{abstract}
Background: Patient preparation for routine abdominal ultrasound, such as fasting for 8 hours and having a light meal the night before the examination, is a common practice employed to avoid digestive motility and gases, which are considered the main causes of artifacts and image quality degradation. Importance of this study: Patient preparation before abdominal ultrasound plays a major role to ensure better visualization of internal organs and pathologies by minimizing artifacts, but because abdominal gases are still present in many patients and in large amounts, causing artifacts, it is important to search for new, safe, efficient, reliable and cost-effective methods to improve patient preparation by eliminating excessive abdominal gases. Material and method: This was a prospective study involving a randomly selected sample of 52 adult patients, both men and women, of different age groups. The participants met the inclusion criteria and had no history of previous or current abdominal surgery. Pediatric patients, pregnant women, patients with a history of abdominal surgery, bedridden patients, and patients with bowel obstruction were excluded. Routine abdominal ultrasound was performed after usual patient preparation involving fasting for 8 hours. Subsequently, the same patients were asked to come again after 48 hours for rescanning and were instructed to follow the same routine preparation and to take one capsule of activated charcoal 8 hours before re-scanning. The results of both scans were compared by focusing on the pancreas as the reference. Result: Significant improvement by $57.2 \%$ was noticed in overweight, obese and extremely obese patients. The improvement noted in underweight patients and in patients with normal weight was $39.05 \%$. The improvement in males reached $66.9 \%$ while in females: $49.9 \%$. The overall improvement in pancreas visualization was $63 \%$. Conclusion: Our preliminary study concluded that activated charcoal can be used to reduce gastrointestinal gases efficiently, providing an inexpensive, safe and easy-to-use method to improve visualization in routine abdominal ultrasound scans in adults.
\end{abstract}




\section{Introduction}

Abdominal ultrasound plays a major role in the diagnosis of patients referred from outpatient clinics, receiving inpatient care and treated at emergency departments. In recent decades, ultrasound has become a cornerstone in diagnosis of many acute and chronic abdominal diseases, such as infections, inflammations, stones, masses, fluid collections and obstructions. The use of ultrasonography as the first-choice examination has become a standard procedure for doctors all over the world since it is available, safe and non-invasive. In routine abdominal ultrasound, patient preparation involving fasting for 8 hours and having a light meal the night before the examination is a common practice to avoid digestive motility and gases that may cause artifacts and degrade image quality. Even though patient preparation before abdominal ultrasound plays a major role in ensuring better visualization of internal organs and pathologies by minimizing artifacts caused by abdominal gases, which makes the scanning process much easier, abdominal gases still exist in many patients and in large amounts, especially in the pancreatic region, causing artifacts. For this reason, it is important to evaluate new, safe, efficient, reliable and cost-effective methods to improve patient preparation in order to eliminate excessive abdominal gases.

\section{Literature review}

Ultrasound is a form of mechanical energy used in medical imaging. Ultrasound waves are produced by the transducer and can travel within the body. They are reflected back from different structures with different attenuation values, thereby creating an image displayed on the monitor after being processed and reconstructed by the computer. Ultrasound is cheap and safe compared to other modalities. It plays a significant role in ruling out many pathologies. All sonographers must manage challenging image artifacts caused by gases present in the gastrointestinal tract. Usually, these are dirty shadowing and reverberation artifacts distal to gases, and they obscure underlying structures and degrade image quality ${ }^{(1)}$. Factors causing excessive intestinal gas include: intestinal obstruction, motility disorders, infections, irritable bowel syndrome, malabsorption as well as psychological and dietary issues. Different drugs and agents are used for gas reduction; they include: beano, simethicone and activated charcoal. Some studies support the use of activated charcoal in the treatment of intestinal gas, while others do not ${ }^{(2)}$. "Activated charcoal is a highly adsorbent powder made from superheated, high surface area, porous particles produced by pyrolysis of organic material. Its extensive surface area is covered with a carbon-based network that also includes functional groups (e.g., carbonyl, hydroxyl) that adsorb chemicals within minutes of contact, preventing gastrointestinal absorption and subsequent toxicity"(3). Many diseases have been treated with activated charcoal, including epilepsy, vertigo, and anthrax. Activated charcoal has recently been used as an antidote for different poisons and as an anti-gas agent. Large doses of activated charcoal administered over a long time have been demonstrated to be very safe and to produce no side effects ${ }^{(4)}$. Higher BMI, abdominal wall thickness and waist circumference are the main factors that can predict the presence of excessive intestinal gas, which impairs the visualization of the upper abdominal structures and pathologies by ultrasound ${ }^{(5)}$. In abdominal ultrasound, the gall bladder must be full and there should be as little gas in the gastrointestinal tract as possible. The intestinal contents (gas and food particles) have a direct impact on the diagnostic value of abdominal ultrasound since they can induce confusing shadows and inconclusive images. All patients undergoing ultrasound examination are always instructed to fast for several hours prior to the scan, mostly for 8 hours, sometimes for as long as 12 hours, to get the full, extended gall bladder. Otherwise the gall bladder will be contracted and difficult to assess. In addition, fasting patients will have less gas in the duodenum and colon. Patient preparation in the form of a two-day low-calorie diet, administration of laxatives and fasting have reported favorable results ${ }^{(6)}$.

Oral simethicone is used as a pre-medication to reduce the amount of gas in the gastrointestinal tract. In the comparison of two protocols of simethicone pre-medication for ultrasound examination, where patients in one group were given a single dose for three days and patients in the other group were administered one single dose of simethicone 1 hour before ultrasound examination, it was found that the single dose administered 1 hour before scanning was superior in terms of the cost and benefit ratio ${ }^{(5)}$. Significant improvement in the visualization of the pancreas and other retroperitoneal structures was reported after the use of simethicone-coated cellulose as an oral contrast agent. This combined agent was used in order to decrease gas-related artifacts from the bowel. Also, it can serve as an effective oral contrast agent for non-fating patients and in emergency cases ${ }^{(7)}$. In Italy, activated charcoal is widely used to reduce intestinal gas and to prepare patients for abdominal US examinations. The results of using activated charcoal and alpha-galactosidase indicate a significant improvement in abdominal ultrasound in the study settings, but more evidence is needed regarding the time and cost of this form of preparation ${ }^{(8)}$.

Our research was conducted to confirm or disprove the efficiency of using activated charcoal to improve the visualization of upper abdominal organs and achieve better image quality in ultrasound of the abdomen.

\section{Methodology}

A prospective research involved a population sample of 52 adult patients and volunteers, including both genders, persons at different age groups, with different body mass indices (BMI) (Tab. 1) and with no history of previous major abdominal surgery or acute illness. Pediatric patients, pregnant women, persons with a history of 
Tab. 1. Body mass index

\begin{tabular}{|l|c|}
\hline \multicolumn{2}{|c|}{ Body Mass Index } \\
\hline Underweight & $<18.5$ \\
\hline Normal weight & $18.5-24.9$ \\
\hline Overweight & $25-29.9$ \\
\hline Obesity & $30-34.9$ \\
\hline Extreme obesity & $>35$ \\
\hline
\end{tabular}

Tab. 2. Gender and age group classification

\begin{tabular}{|l|c|c|c|c|c|c|}
\hline \multirow{2}{*}{ Sex } & \multicolumn{7}{|c|}{ Age Group } \\
\cline { 2 - 7 } & $\mathbf{1 6 - 2 5}$ & $\mathbf{2 6 - 3 5}$ & $\mathbf{3 6 - 4 5}$ & $\mathbf{4 6 - 5 5}$ & $\mathbf{5 5 - 6 5}$ & Total \\
\hline Males & 3 & 7 & 7 & 3 & 3 & 23 \\
\hline Females & 6 & 3 & 2 & 4 & 2 & 17 \\
\hline Total & 9 & 10 & 9 & 7 & 5 & 40 \\
\hline
\end{tabular}

Tab. 3. Body mass index classification

\begin{tabular}{|l|c|c|c|c|c|}
\hline \multirow{2}{*}{ Sex } & \multicolumn{5}{|c|}{ Body Mass Index } \\
\cline { 2 - 6 } & Underweight & $\begin{array}{c}\text { Normal } \\
\text { weight }\end{array}$ & Overweight & Obesity & $\begin{array}{c}\text { Extreme } \\
\text { obesity }\end{array}$ \\
\hline Male & 0 & 8 & 4 & 8 & 3 \\
\hline Female & 2 & 5 & 4 & 3 & 3 \\
\hline Total & 2 & 13 & 8 & 11 & 6 \\
\hline
\end{tabular}

abdominal surgery, bedridden patients, and patients with bowel obstruction where excluded. The Ethics Committee of King Abdulaziz University approved the study protocol. Informed consent was obtained from all patients and volunteers. The routine upper abdominal ultrasound was preceded with usual preparation involving fasting for 8 hours prior to the examination (only water was allowed). Each patient was scanned twice; the first scan was conducted after the routine preparation, while the second scan was conducted after modified preparation where the same patient was instructed to keep fasting and additionally take one charcoal capsule orally 8 hours prior to the examination. Subsequently, we compared image quality using the pancreas as the reference. Ultrasound images obtained in both examinations from each patient were evaluated by a qualified radiologist and a senior sonographer using a specially designed evaluation form.

\section{Results}

A sample size of 52 patients and volunteers included both genders. Twelve of them were excluded and only 40 candidates were included: 23 men and 17 women at the age range from 18 to 61 years, and with different BMI values (Tab. 1, Tab. 2, Tab. 3).

The images obtained before and after administration of an activated charcoal capsule were evaluated by two physicians experienced in radiology at King Abdulaziz University Hospital (KAUH): body-imaging radiologist and a senior sonographer with a PHD degree in ultrasound. They evaluated and classified the images into three categories: very good, good and poor. As for images of the head of the pancreas obtained before charcoal administration, $10 \%$ were classified as very good, $47.5 \%$ as good and $42.5 \%$ as poor. The results of the neck of the pancreas were as follows: $33.75 \%$ of very good images, $42.5 \%$ of good images and $28.75 \%$ of poor images. The images of the body of the pancreas were very good for $15 \%$ of images, good for $33.75 \%$ of images and poor for $51.2 \%$ of images. As for the tail of the pancreas, there were 0 very good images, $21.2 \%$ of good images and $78.75 \%$ of poor images, which was the highest result recoded (Fig. 1). The mean values regarding the visualization of the head of the pancreas after charcoal administration were: $35 \%$ in the very good group, $45 \%$ in the good group and $20 \%$ in the poor group. The results for the neck of the pancreas were as follows: $45 \%$ of very good images, $45 \%$ of good images and $10 \%$ of poor images. The images of the body of the pancreas were very good in $17.5 \%$ of cases, good in $60 \%$ of cases and poor in $22.5 \%$ of cases. The respective values for the tail of the pancreas were: $7.5 \%, 30 \%$ and $62.5 \%$ (Fig. 2).

\section{Head of the pancreas}

The results regarding the visualization of the head of the pancreas in 40 examined patients changed from $10 \%$ before charcoal administration to $35 \%$ after charcoal administration in the very good group, from $47.5 \%$ to $45 \%$ in the good group, and from $42.5 \%$ to $20 \%$ in the poor group, respectively; therefore the overall improvement was $50 \%$.

\section{Neck of the pancreas}

As for the neck of the pancreas, the results were as follows: $33.75 \%$ and $45 \%$ of very good images, $42.5 \%$ and $45 \%$ of good images, and $28.75 \%$ and $10 \%$ of poor images,

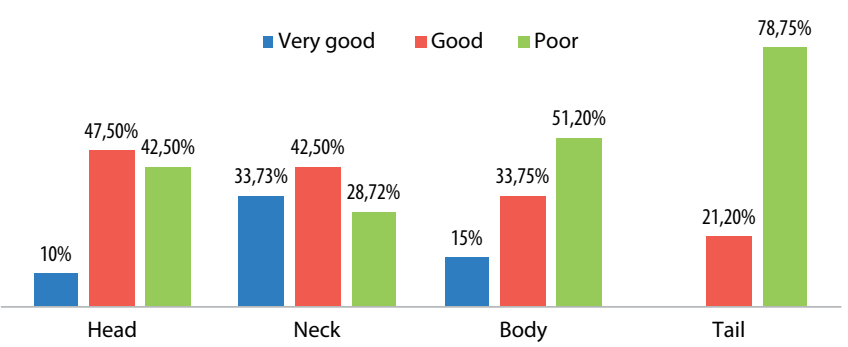

Fig. 1. Average values from two evaluations before activated charcoal administration

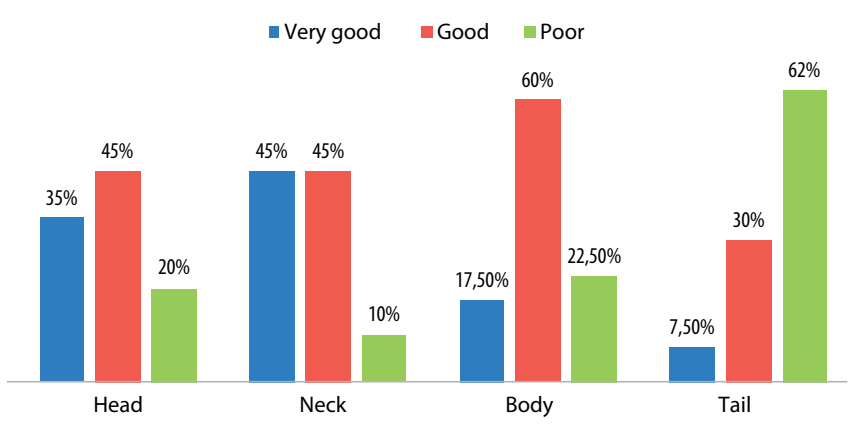

Fig. 2. Average values from two evaluations after activated charcoal administration 
respectively for images obtained before and after charcoal administration. The overall improvement was $32.5 \%$.

\section{Body of the pancreas}

For the body of the pancreas, the results were as follows: $15 \%$ and $17.5 \%$ in the very good group, $33.75 \%$ and $60 \%$ for the good group, and $51.2 \%$ and $22.5 \%$ for the poor group, respectively for images obtained before and after charcoal administration. The overall improvement was therefore $57.45 \%$.

\section{Tail of the pancreas}

There were 0 and $7.5 \%$ of very good images, $21.2 \%$ and $30 \%$ of good images, and $78.75 \%$ and $62.5 \%$ of poor images, respectively for images obtained before and after charcoal administration. The overall improvement was therefore $32.55 \%$.

\section{Overall evaluation of image quality after administration of activated charcoal}

The mean result obtained by both evaluators in terms of the overall criteria (much better, better, no difference and worse) was $21 \%$ for "much better," $42 \%$ for "better," $33 \%$ for "no difference" and only $4 \%$ for "worse." The overall improvement in pancreas visualization was therefore $63 \%$ in all 40 cases (Fig. 3).

\section{Improvement in relation to body mass index}

The overall improvement of $57.2 \%$ (much better and better) was noted for overweight, obese and extremely obese patients, whereas the value of only $39.05 \%$ was noted for underweight patients and for patients with normal body weight. In overweight, obese and extremely obese males the overall improvement reached $66.9 \%$ and the respective value for females was $49.9 \%$ (Tab. 4).

\section{No improvement}

The overall value for no improvement (no difference) was $62.4 \%$ in overweight, obese and extremely obese patients
Tab. 4. Improvement in two evaluations in relation to BMI and gender

\begin{tabular}{|l|c|c|c|}
\hline Result & $\begin{array}{c}\text { Body } \\
\text { radiologist }\end{array}$ & $\begin{array}{c}\text { Senior- } \\
\text { sonographer }\end{array}$ & $\begin{array}{c}\text { Mean } \\
\text { value }\end{array}$ \\
\hline $\begin{array}{l}\text { Improvement (overweight, } \\
\text { obese, extremely obese) }\end{array}$ & $53.3 \%$ & $61.1 \%$ & $57.2 \%$ \\
\hline $\begin{array}{l}\text { Improvement (underweight } \\
\text { and normal weight) }\end{array}$ & $40 \%$ & $38.09 \%$ & $39.045 \%$ \\
\hline Improvement in males & $58.8 \%$ & $75 \%$ & $66.9 \%$ \\
\hline Improvement in females & $46 \%$ & $53.8 \%$ & $49.9 \%$ \\
\hline
\end{tabular}

and only $37.2 \%$ for underweight patients and for patients with normal weight. In overweight, obese and extremely obese males the "no difference" value reached $36.9 \%$, and the respective value for females was $62.5 \%$ (Fig. 4).

\section{Relationship of BMI with the individual categories}

The mean results from both analyses in relation to the BMI were as follows: the "much better" category: $12 \%$ in underweight patients, $29 \%$ in patients with normal weight, $29 \%$ in overweight patients, $18 \%$ in obese patients and $12 \%$ in extremely obese patients; the "better" category: $3 \%$ in underweight patients, $35 \%$ in patients with normal weight, $29 \%$ in overweight patients, $21 \%$ in obese patients and $12 \%$ in extremely obese patients; the "no difference" category: $4 \%$ in underweight patients, $35 \%$ in patients with normal weight, $4 \%$ in overweight patients, $38 \%$ in obese patients and $19 \%$ in extremely obese patients; the "worse" category: $67 \%$ in obese patients and $33 \%$ in extremely obese patients (Fig. 5).

The mean results from both analyses in relation to the gender were as follows: the "much better" category: $11 \%$ in males and 35\% in females; the "better" category: $43 \%$ in males and $41 \%$ in females; the "no difference" category: $39 \%$ in males and $24 \%$ in females; the "worse" category $7 \%$ in males and 0 in females (Fig. 6).

\section{Relationship with age group}

The results for the "much better" category were as follows: $41 \%$ in age group 16-25 years (the highest value), $23 \%$ in age group $36-45$ years and $18 \%$ in two age groups: $26-35$

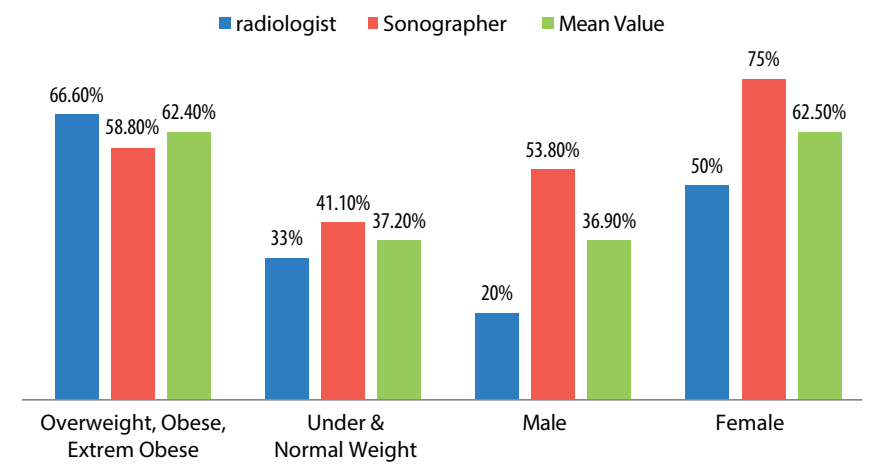

Fig. 4. Images that were not improved in relation to BMI and gender 


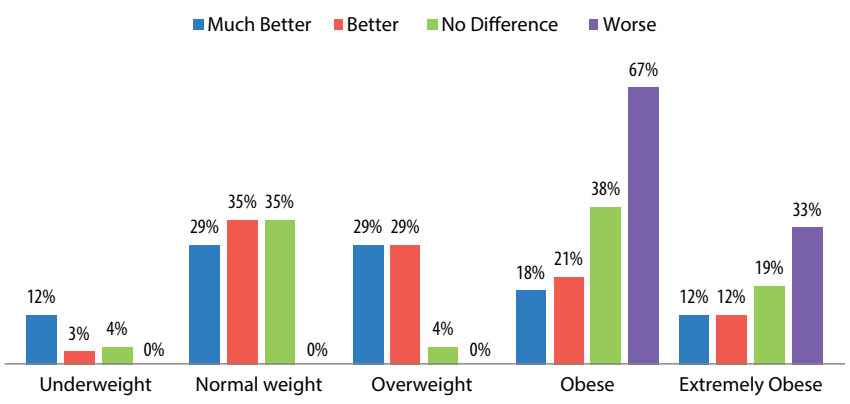

Fig. 5. Average values from two evaluations for individual categories in relation to $B M I$

years and 46-55 years. For the "better" category, the following values were noted: $29 \%$ (the highest value) in age group 36-45 years, $26 \%$ in age group 26-35 years, $21 \%$ in age group 16-25 years and 12\% in two age groups 26-35 years and 46-55 years. The highest result in the "no difference" category was $31 \%$ in age group 26-35 years, followed by $23 \%$ in age group 56-65 years, $19 \%$ in age group 46-55 years, $15 \%$ in age group 16-25 years and only $12 \%$ in age group 36-45 years The "worse" category was selected only in two age groups: $33 \%$ in age group 36-45 years and 66\% in age group $46-55$ years.

\section{Discussion}

Activated charcoal is a highly adsorbent powder made from superheated, high surface area, porous particles produced from organic material ${ }^{(3)}$. It is an excellent adsorbent of many chemical substances, including gases ${ }^{(9)}$. The unsatisfactory ultrasound scans of the pancreas are frequently caused by an excessive amount of abdominal gases. This study showed that activated charcoal can improve pancreas visualization in ultrasound. The results mentioned above indicate that considerable improvement concerned the head of the pancreas and the body of the pancreas, where the highest improvement rates were obtained. The total improvement in the visualization of the pancreatic head was $50 \%$, which was expected since the head of the pancreas is easy to access compared to other parts of the pancreas. Administration of activated charcoal to patients undergoing abdominal ultrasound contributed to a noticeable reduction of gases, which led to an increase in the

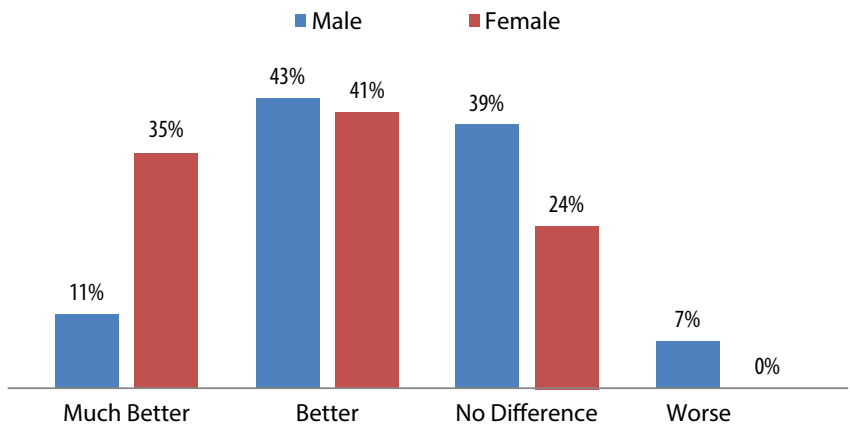

Fig. 6. Average values from two evaluations for individual categories in relation to gender quality of the visualization of the pancreatic head. As for the neck of the pancreas, the improvement in visualization was also observed (32.5\%) for images obtained after charcoal administration compared to pre-administration scans.

Moreover, the study showed that the greatest improvement concerned the visualization of the body of the pancreas compared to the rest of the organ, where the overall improvement was $57.45 \%$. The tail of the pancreas is usually very difficult to visualize with routine preparation for abdominal ultrasound because of its location between the stomach and the spleen. In this case, image quality improved by $32.5 \%$ after activated charcoal administration.

We found that significant overall improvement was related to BMI; in overweight, obese and extremely obese patients, improvement of image quality reached $57.2 \%$, which is higher than the result for underweight patients and in patients with normal weight (only 39.05\%). The improvement of image quality after charcoal administration in males was $66.9 \%$ while in females $49.9 \%$, as shown in Tab. 4. On the other hand, the "not improved" images in males were found for $36.9 \%$ of cases. The respective value for females was $62.5 \%$, and this had a negative effect on the overall improvement in females, which was $49.9 \%$, as shown in Fig. 4. A low level of physical activity in females as seen in our society may be the major factor that affects the efficiency of activated charcoal in women. From our study, we concluded that adding one capsule of activated charcoal to the patient preparation routine in a sample of 40 patients directly improved the visualization of the pancreas by $63 \%$. Image quality was not improved in $37 \%$ of the cases, as shown in Fig. 3.

\section{Conclusion}

Our preliminary study concluded that activated charcoal is useful, safe and cost-effective, and can be used to reduce gastrointestinal gases and improve the visualization of the pancreas and other structures in routine abdominal ultrasound in adults. Patient preparation for a routine abdominal ultrasound examination can be updated by adding an activated charcoal capsule to eliminate gases that usually degrade image quality in abdominal ultrasonography.

\section{Limitations}

- Limited time to complete the research

- The sample size was not large as patients refused participation in this research as additional preparation and a re-scan were needed.

\section{Recommendations}

- It is recommended to conduct further investigation with an increased dose to two capsules of activated charcoal instead of one.

- The sample size should be increased in future research for both genders. 
- It is recommended to fine-tune patient preparation by instructing patients to restrain from having fizzy drinks and avoid heavy meals for two days before the examination in addition to administration of activated charcoal.

- Patient instructions should be revised and the statement "avoid milk, coffee and smoking on the day of the examination" should be added.

\section{References}

1. Wilson SR, Burns PN, Wilkinson LM, Simpson DH, Muradali D: Gas at abdominal US: appearance, relevance, and analysis of artifacts. Radiology 1999; 210: 113-123, DOI: 10.1148/radiology.210.1.r99ja12113.

2. Abraczinskas D, Goldfinger S: Intestinal gas and bloating. UpToDate 2013. Topic 2607, version 7. Online: http://ultramedica.net/Uptodate21.6/contents/UTD.htm?24/18/24872?source=related_link\#H23 [dostęp 13.01.2018].

3. Hendrickson R, Kusin S: Gastrointestinal decontamination of poisoned adults. UpToDate 2013. Topic 321, version 10. Online: http://ultramedica.net/Uptodate21.6/contents/UTD.htm?15/58/16297?source=see_link [dostęp 13.01.2018].

4. Jain NK, Patel VP, Pitchumoni CS: Efficacy of activated charcoal in reducing intestinal gas: a double-blind clinical trial. American Journal of Gastroenterology 1986; 81: 532-535. Online: https://ncbi.nlm.nih.gov/ pubmed/3521259.

5. Marsico M, Gabbani T, Casseri T, Biagini MR: Factors predictive of improved abdominal ultrasound visualization after oral administration of simethicone. Ultrasound Med Biol 2016; 42: 2532-2537. Online: http:// sciencedirect.com/science/article/pii/S0301562916301557.

\section{Conflicts of interest}

Authors do not report any financial or personal connections with other persons or organizations, which might negatively affect the contents of this publication and/or claim authorship rights to this publication.
6. Vogel HJ, Schipper J, Hermans J: Abdominal ultrasonography: improved image quality with the combined use of a diet and laxatives. J Clin Ultrasound 1990; 18: 627-630. Online: https://ncbi.nlm.nih.gov/ pubmed/2172309.

7. Harisinghani MG, Saini S, Schima W, McNicholas M, Mueller P: Simethicone coated cellulose as an oral contrast agent for ultrasound of the upper abdomen. Clin Radiol 1997; 52: 224-226. Online: http:// clinicalradiologyonline.net/article/S0009-9260(97)80277-2/pdf .

8. Maconi G, Bolzacchini E, Radice E, Marzocchi M, Badini M: Alphagalactosidase versus active aharcoal for improving sonographic visualization of abdominal organs in patients with excessive intestinal gas. J Ultrasound 2012; 15: 232-238. Online: http://sciencedirect.com/science/ article/pii/S197134951200032X?via\%3Dihub\#!.

9. Hall RG Jr, Thompson H, Strother A: Effects of orally administered activated charcoal on intestinal gas. Am J Gastroenterol 1981; 75: 192-196. Online: https://www.researchgate.net/publication/16182886_Effects_of_ orally_administered_activated_charcoal_on_intestinal_gas. 